\title{
Abstract: Defence of Mathematical Models for Deep Learning based Registration
}

\author{
Lasse Hansen, Maximilian Blendowski, Mattias P. Heinrich \\ Institut für Medizinische Informatik, Universität zu Lübeck \\ hansen@imi.uni-luebeck.de
}

Deep learning based methods have not reached clinically acceptable results for common medical registration tasks that could be adequately solved using conventional methods. The slower progress compared to image segmentation is due to the lower availability of expert correspondences and the very large learnable parameter space for naive deep learning solutions. We strongly believe that it is necessary and beneficial to integrate conventional optimisation strategies as differentiable modules into deep learning based registration. The process can then be broken down into smaller components (e.g. feature learning and regularisation) that are easier to train and enable explainable network models. In [1], we propose to learn interpretable multi-modal features that can be directly employed using classical iterative registration schemes. The differentiable operations within a supervised descent approach enables us to learn a mapping of the complex multi-modal appearance to a common space. Incorporating keypoint models is vital for state-of-the-art performance in challenging $3 \mathrm{D}$ thoracic registration. Deep learning of sparse point cloud matching using graph convolutions was shown in [2] to predict keypoint features and correspondences solely by their inherent geometric structure. Approximate mean-field inference (based on a graphical model) together with densely sampled discrete displacements has been shown to outperform previous work on abdominal CT registration and enabling the learning of large deformation with few labelled scans [3]. Our work demonstrates advantages over state-of-the-art fully-convolutional models and provides an interesting avenue for further research. ${ }^{1}$

\section{References}

1. Blendowski M, Heinrich MP. Learning interpretable multi-modal features for alignment with supervised iterative descent. In: MIDL; 2019. p. 73-83.

2. Hansen L, Dittmer D, Heinrich MP. Learning deformable point set registration with regularized dynamic graph CNNs for large lung motion in COPD patients. arXiv preprint arXiv:190907818. 2019;.

3. Heinrich MP. Closing the gap between deep and conventional image registration using probabilistic dense displacement networks. In: MICCAI; 2019. p. 50-58.

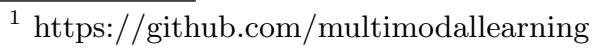

